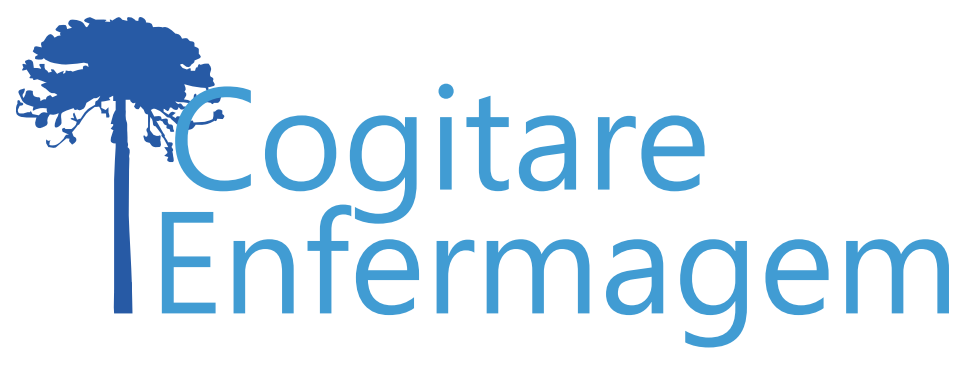

\title{
HANSENÍASE EM PAÍSES FRONTEIRIÇOS NA AMÉRICA DO SUL: UM ESTUDO ECOLÓGICO*
}

Keurilene Sutil de Oliveira1, Marcos Augusto Moraes Arcoverde ${ }^{2}$, Enrique Jorge Deschutter ${ }^{3}$, Alex Junior da Silva ${ }^{4}$, Adriana Zilly ${ }^{5}$, Reinaldo Antonio da Silva Sobrinho ${ }^{6}$

\section{RESUMO}

Objetivo: caracterizar o perfil clínico epidemiológico e a distribuição espacial da incidência da hanseníase em territórios fronteiriços da América do Sul.

Método: trata-se de um estudo ecológico. O estudo compreendeu a Província de Misiones na Argentina e a Região Sul do Brasil. A população foi composta por 10.319 casos novos de hanseníase, diagnosticados entre 2010 e 2016.

Resultados: o estado do Paraná foi o mais endêmico, representando $70,2 \%(n=7,247)$ dos casos. Houve predomínio da classificação operacional multibacilar $(79,8 \%, n=8.233)$ e Grau 0 de incapacidade física $(50,6 \%, n=5.223)$. Em Misiones 18,9\% utilizaram esquema de tratamento substitutivo. No período, notou-se uma situação hiperendêmica em 780 (65,5\%) dos municípios/ departamentos estudados.

Conclusão: o estudo mostrou que as regiões estudadas apresentam alta endemicidade, transmissão ativa e diagnóstico tardio da hanseníase. Essas tendências entrelaçadas à força de morbidade e de transmissão recente e persistente da doença, ampliam a relevância da hanseníase como problema de saúde pública na região.

DESCRITORES: Hanseníase; Incidência; Análise espacial; Saúde na fronteira; Doenças transmissíveis.

*Artigo extraído da dissertação de mestrado "Distribuição espacial e temporal da hanseníase na Província de Misiones-Argentina e Região Sul do Brasil". Universidade Estadual do Oeste do Paraná, 2019.

COMO REFERENCIAR ESTE ARTIGO:

Oliveira KS de, Arcoverde MAM, Deschutter JH, Silva AJ da, Zilly A, Silva Sobrinho RA da. Hanseníase em países fronteiriços na América do Sul: um estudo ecológico. Cogitare enferm. [Internet]. 2019 [acesso em "colocar data de acesso, dia, mês abreviado e ano"]; 24. Disponível em: http://dx.doi.org/10.5380/ce.v24i0.64917.

Este obra está licenciado com uma Licença Creative Commons Atribuição 4.0 Internacional.

${ }^{1}$ Enfermeira. Mestranda em Saúde Pública em região de Fronteira. Enfermeira na Prefeitura Municipal de São Miguel do Iguaçu. São Miguel do Iguaçu, PR, Brasil.

Enfermeiro. Doutor em Saúde Pública. Docente da Universidade Estadual do Oeste do Paraná. Foz do Iguaçu, PR, Brasil. () ${ }^{3}$ Bioquímico. Doutor em Saúde Pública. Diretor e docente da Maestría Salud Pública y Enfermedades Transmisibles. Universidade Nacional de Misiones. Posadas, Misiones, Argentina.

${ }^{4}$ Matemático. Especialista em Metodologia do Ensino da Matemática e da Física. Universidade Estadual do Oeste do Paraná. Foz do Iguaçu, PR, Brasil. (0)

${ }^{5}$ Bióloga. Doutora em ciências biológicas. Docente da Universidade Estadual do Oeste do Paraná. Foz do lguaçu, PR, Brasil. (?) ${ }^{6}$ Enfermeiro. Pós-doutor em Saúde Pública. Coordenador e docente do programa de pós-graduação (Stricto Sensu) em Saúde pública em Região de Fronteira da Universidade Estadual do Oeste do Paraná. Foz do Iguaçu, PR, Brasil. 


\title{
LEPROSY IN BORDER COUNTRIES IN SOUTH AMERICA: AN ECOLOGICAL STUDY
}

\begin{abstract}
Objective: to characterize the epidemiological clinical profile and spatial distribution of the incidence of leprosy in border territories of South America.

Method: this is an ecological study. The study included the Province of Misiones in Argentina and the Southern Region of Brazil. The population consisted of 10,319 new leprosy cases diagnosed between 2010 and 2016.

Results: the state of Paraná was the most endemic, representing 70.2\% ( $(n=7.247)$ of the cases. There was a predominance of multibacillary operational classification $(79.8 \%, n=8,233)$ and Grade 0 of physical disability (50.6\%, n=5,223). In Misiones, $18.9 \%$ used a substitution treatment regimen. In the period, a hyperendemic situation was observed in $780(65.5 \%)$ of the studied cities/departments.

Conclusion: the study revealed that the regions studied have high endemicity, active transmission and late diagnosis of leprosy. These intertwined trends in the strength of morbidity and recent and persistent transmission of the disease increase the relevance of leprosy as a public health problem in the region.
\end{abstract}

DESCRIPTORS: Leprosy; Incidence; Spatial analysis; Border health; Transmissible diseases.

\section{LEPRA EN LOS TERRITORIES FRONTERIZOS DE SUDAMÉRICA: ESTUDIO ECOLÓGICO}

\begin{abstract}
RESUMEN:
Objetivo: caracterizar el perfil clínico epidemiológico y la distribución espacial de la incidencia de la lepra en los territorios fronterizos de Sudamérica.

Metodología: se trata de un estudio ecológico. El estudio incluyó la Provincia de Misiones en Argentina y la Región Sur de Brasil. La población estaba compuesta por 10.319 nuevos casos de lepra diagnosticados entre 2010 y 2016.

Resultados: el estado de Paraná fue el más endémico, representando el 70,2\% ( $n=7.247)$ de los casos. Predominaron la clasificación operacional multibacilar $(79,8 \%, n=8.233)$ y el Grado 0 de discapacidad física $(50,6 \%, n=5.223)$. En Misiones, el 18,9\% utilizó un régimen de tratamiento de sustitución. En el periodo se observó una situación hiperendémica en 780 $(65,5 \%)$ de las ciudades/departamentos estudiados.

Conclusión: El estudio mostró que las regiones estudiadas tienen alta endemicidad, transmisión activa y diagnóstico tardío de la lepra. Estas tendencias entrelazadas en cuanto a la fuerza de la morbilidad y la transmisión reciente y persistente de la enfermedad aumentan la importancia de la lepra como un problema de salud pública en la región.
\end{abstract}

DESCRIPTORES: Lepra; Incidencia; Análisis Espacial; Salud Fronteriza; Enfermedades Transmisibles. 
O Mycobacterium leprae é o bacilo agente etiológico causador da doença infecciosa crônica, denominada hanseníase, o qual infecta macrófagos e células de Schwann ${ }^{(1)}$. São várias as manifestações clínicas, sendo mais predominantes as lesões de pele com sensibilidade diminuída e nervos subcutâneos espessados ${ }^{(2)}$.

A porta de entrada do bacilo são as vias aéreas superiores e ocorre por transmissão direta ${ }^{(3)}$, e devido às graves incapacidades físicas permanentes, que levam a sérias limitações na realização de atividades diárias, a hanseníase é considerada um problema de saúde pública mundial(4).

Os casos de hanseníase no Brasil diminuíram $26 \%$ entre 2001 e $2011^{(5)}$. No ano de 2014, a prevalência foi de 1,2/10.000 habitantes, correspondendo a 25.738 casos em tratamento. No mesmo ano, o Brasil apresentou coeficiente de detecção geral de casos novos de 15,3/100.000 habitantes, o que corresponde a 31.064 casos novos da doença, apresentando classificação de alta endemicidade, segundo parâmetros oficiais ${ }^{(6)}$. No ano de 2017, o total de casos novos de hanseníase no Brasil foi de 22.940, dos quais 1.718 foram em menores de 15 anos, indicando transmissão ativa da doença ${ }^{(4)}$.

Na Argentina, o número de casos novos de hanseníase em 2010 foi de 354, subindo em 2015 para 586 casos. A hanseníase é endêmica nas províncias do noroeste, nordeste e centro do país: Chaco, Formosa, Corrientes, Misiones, Santa Fe, Entre Rios, Córdoba, Santiago del Estero, Tucumán, Salta, Jujuy, Buenos Aires e Capital Federal ${ }^{(7)}$.

A distribuição da doença não é uniforme na Argentina, no Sul do país a reduzida notificação de casos novos parece relacionada com migração de trabalhadores temporários. Entretanto, projeções indicam uma tendência sustentada de aumento nas proporções de casos multibacilares (MB) e com grau dois de incapacidade física. A maioria dos casos concentra-se no norte do país, justamente em espaços territoriais próximos à fronteira com - Paraguai e Brasil onde o nível de endemicidade é elevado, tais aspectos demonstram que há circulação bacilar ativa e risco de aparecimento de casos novos ${ }^{(8)}$.

Todos os anos mais de 200.000 novos casos de hanseníase são registrados no mundo, em consequência, a Organização Mundial da Saúde (OMS) estabeleceu um objetivo para interromper a transmissão da hanseníase em todo o mundo até 2020, destacando a necessidade de medir a carga da doença de forma mais abrangente ${ }^{(9)}$, incluindo promover parcerias intersetoriais no âmbito internacional e nacional. Até mesmo países com poucos doentes, como é o caso da Argentina, devem manter serviços de vigilância e referência, sobretudo em razão da migração de países onde a hanseníase ainda é endêmica ${ }^{(10)}$.

O risco de transmissão da hanseníase e a influência da situação epidemiológica em regiões vizinhas ${ }^{(11)}$ mostram a importância de estudos analíticos espaciais para identificar áreas geográficas em que intervenções específicas podem ser realizadas para o controle da doença ${ }^{(12)}$. Destacando as regiões de fronteiras, onde há carências de estudos e fragilidade em vigiar a migração de populações entre os diferentes países ${ }^{(11)}$.

Diante do exposto, este estudo tem como objetivo caracterizar o perfil clínico epidemiológico e a distribuição espacial da incidência da hanseníase em territórios fronteiriços da América do Sul.

\section{MÉTODO}

Trata-se de um estudo epidemiológico do tipo ecológico. O estudo compreendeu a Província de Misiones na Argentina e a Região Sul do Brasil, representados na Figura 1. 


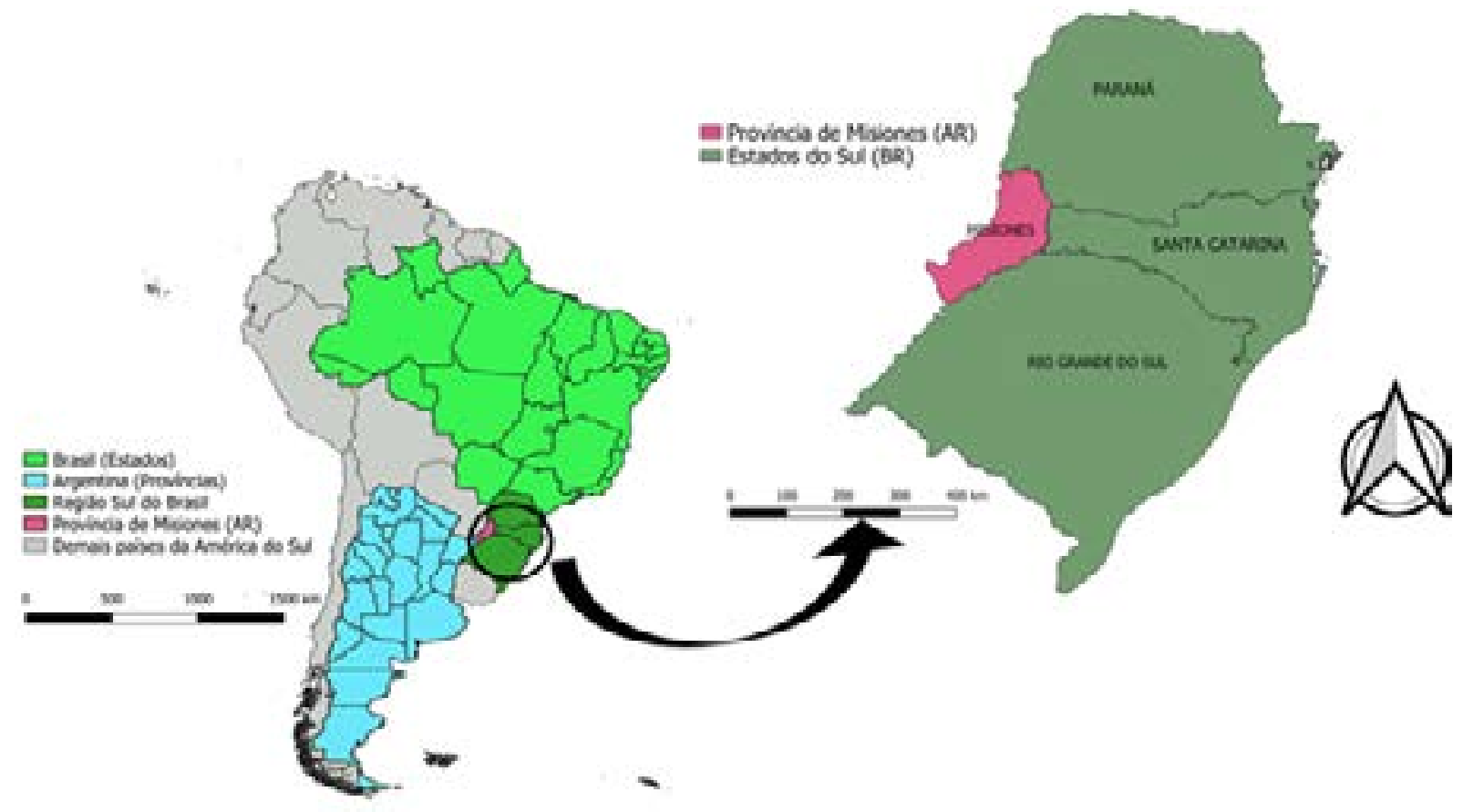

Figura 1 - Localização geográfica da Região Sul do Brasil e Província de Misiones Argentina. Província de Misiones (AR), Paraná (BR), Santa Catarina (BR), Rio Grande do Sul (BR), 2010 a 2016

A Argentina é composta por 23 províncias e a capital denominada cidade autônoma de Buenos Aires, cada ente possui constituição própria, mas convivem em um sistema federal. As províncias são divididas em departamentos, exceto a província de Buenos Aires, que é dividida em partidos ${ }^{(13)}$.

A Província de Misiones está localizada na Região Nordeste do país, portanto, faz parte de uma das regiões endêmicas para hanseníase. Quase todos os seus limites territoriais são constituídos por rios e mais de $80 \%$ são fronteiras internacionais, a Norte e Leste com - Brasil, ao Oeste com Paraguai e ao sul com a província de Corrientes (Argentina). É organizada politicamente em 17 departamentos, divididos em 75 municípios. A área total é de aproximadamente $29.801 \mathrm{~km}^{2}$, e no ano de 2014 a população era de 1.175 milhões ${ }^{(14)}$.

Neste estudo utilizamos os departamentos de Misiones como unidade de análise, pois a população dos municípios mostrou-se pequena para comparação com os municípios brasileiros, além de que, na Argentina, a unidade municipal é política e administrativamente diferente do Brasil, o que reforça o uso de departamentos como unidade de análise no país vizinho.

O setor saúde da Argentina é estruturado com base em três subsetores principais: Subsetor público com financiamento e provisão público, Subsetor de seguro social obrigatório e, por último, o Subsetor privado ${ }^{(15)}$.

A Região Sul do Brasil é constituída por três estados: Paraná, Santa Catarina e Rio Grande do Sul. O Paraná, no ano 2016, teve sua população estimada em 11.242.720 milhões de habitantes que residem nos 399 municípios, e sua área é de aproximadamente $199.307,939 \mathrm{~km}^{2}$. Santa Catarina possui 295 municípios e um território de $112.872 \mathrm{~km}^{2}$, e sua população estimada em 2014 foi de 6.727 milhões. O estado do Rio Grande do Sul é dividido em 497 municípios, sua área total é de 281.730,223 km² e a população em 2014 era de 11.207 milhões de habitantes ${ }^{(16)}$.

A população de estudo foi composta por 10.319 casos novos de hanseníase (Classificação Estatística Internacional de Doenças e problemas Relacionados à Saúde CID 10 de A30.0 a A30.9) que foram diagnosticados no período de 2010 a 2016 nos 
três estados da região sul do Brasil e na província de Misiones na Argentina. O período analisado foi escolhido por ser o período em que se encontraram dados completos para todas as variáveis eleitas para análise em ambos os países.

A busca de dados foi realizada no primeiro semestre de 2018. Para a Região Sul do Brasil, os dados foram solicitados pelo Sistema Eletrônico do Serviço de Informação ao cidadão (e-SIC) fornecido pelo Ministério da Saúde - Brasil. Quanto à Província de Misiones, os dados foram obtidos através do Ministério de Salud de Misiones-Argentina.

Para análise descritiva utilizou-se as variáveis sexo, classificação operacional, grau de incapacidade física e esquema de tratamento. Para a construção da taxa de incidência padronizada da hanseníase por 100 mil habitantes, lançou-se mão da variável casos novos notificados e a população residente segundo território. A taxa de incidência foi padronizada de forma direta considerando idade e sexo.

Na análise descritiva, os dados foram apresentados em frequência absoluta e relativa. Para a autocorrelação espacial, utilizou-se como ferramenta estatística o Índice Moran Global e Local (Índice Local de Associação Espacial - LISA). O padrão de distribuição da incidência da hanseníase foi examinado em menor escala por meio do Moran local, produzindo um valor específico para cada município/departamento, permitindo a visualização de agrupamentos com associações espaciais significativos. Essa análise foi realizada apenas no território brasileiro devido à incompatibilidade na união dos arquivos de projeção geográfica. Para esta análise estatística foi considerado o valor de significância de $5 \%$.

A pesquisa seguiu os preceitos éticos do Conselho Nacional Saúde e Declaração de Helsinki da Associação Médica Mundial, e todas determinações da Resolução 466/2012 do Conselho Nacional de Saúde. O estudo obteve aprovaçã̃o da instituição de saúde e do Comitê de Ética em Pesquisa envolvendo Seres Humanos, sendo o número do parecer 2.588.573.

\section{RESULTADOS}

Foram registrados 10.319 casos novos de hanseníase na Região Sul do Brasil e na Província de Misiones, Argentina. O estado do Paraná foi o mais endêmico, com 7,247 $(70,2 \%)$ casos registrados. Do total, foram identificados $6.082(59 \%)$ casos do sexo masculino. Houve predomínio da classificação operacional multibacilar 8.233 (79,8\%) e Grau 0 de incapacidade física $5.223(50,6 \%)$. Porém, 468 (4,5\%) pacientes não foram avaliados quanto ao grau de incapacidade física (Tabela 1 ).

Tabela 1 - Distribuição dos casos novos de hanseníase diagnosticados segundo sexo, classificação operacional e grau de incapacidade física. Província de Misiones (AR), Paraná (BR), Santa Catarina (BR), Rio Grande do Sul (BR), 2010 a 2016 (continua)

\begin{tabular}{|c|c|c|c|c|c|c|c|c|c|c|c|}
\hline & & \multicolumn{2}{|c|}{ MISIONES } & \multicolumn{2}{|c|}{ PARANÁ } & \multicolumn{2}{|c|}{$\begin{array}{c}\text { SANTA } \\
\text { CATARINA }\end{array}$} & \multicolumn{2}{|c|}{$\begin{array}{c}\text { RIO GRANDE } \\
\text { DO SUL }\end{array}$} & \multicolumn{2}{|c|}{ TOTAL } \\
\hline & & $\mathbf{N}$ & $\%$ & $\mathbf{n}$ & $\%$ & $\mathbf{n}$ & $\%$ & $\mathbf{n}$ & $\%$ & $\mathbf{n}$ & $\%$ \\
\hline \multirow[t]{2}{*}{ SEXO } & Feminino & 118 & 43,7 & 2916 & 40,2 & 640 & 40,6 & 563 & 46 & 4237 & 41 \\
\hline & Masculino & 152 & 56,3 & 4331 & 59,8 & 937 & 59,4 & 662 & 54 & 6082 & 59 \\
\hline \multirow{2}{*}{$\begin{array}{l}\text { CLASSIFICAÇÃO } \\
\text { OPERACIONAL }\end{array}$} & Paucibacilar & 40 & 14,8 & 1462 & 20,2 & 370 & 23,5 & 214 & 17,5 & 2086 & 20,2 \\
\hline & Multibacilar & 230 & 85,2 & 5785 & 79,8 & 1207 & 76,5 & 1011 & 82,5 & 8233 & 79,8 \\
\hline
\end{tabular}




\begin{tabular}{llcccccccccc}
\hline $\begin{array}{l}\text { GRAU DE } \\
\text { INCAPACIDADE } \\
\text { FÍSICA }\end{array}$ & Grau 0 & 155 & 57,4 & 3754 & 51,8 & 799 & 50,7 & 515 & 42 & 5223 & 50,6 \\
\cline { 2 - 12 } & Grau I & 103 & 38,2 & 2461 & 34 & 472 & 29,9 & 390 & 31,8 & 3426 & 33,2 \\
\cline { 2 - 12 } & Grau II & 12 & 4,4 & 810 & 11,2 & 193 & 12,2 & 187 & 15,3 & 1202 & 11,7 \\
\cline { 2 - 11 } & Não Avaliado & - & - & 222 & 3 & 113 & 7,2 & 133 & 10,9 & 468 & 4,5
\end{tabular}

Em relação ao tipo de tratamento, 7.539 (73\%) fizeram uso da poliquimioterapia (PQT) MB e $2.099(20,4 \%)$ PQT paucibacilar (PB). Na província de Misiones 51 (18,9\%) casos utilizaram esquema de tratamento substitutivo (Tabela 2 ).

Tabela 2 - Distribuição dos casos de hanseníase, segundo esquema de tratamento. Província de Misiones $(A R)$, Paraná (BR), Santa Catarina (BR), Rio Grande do Sul (BR), 2010 a 2016

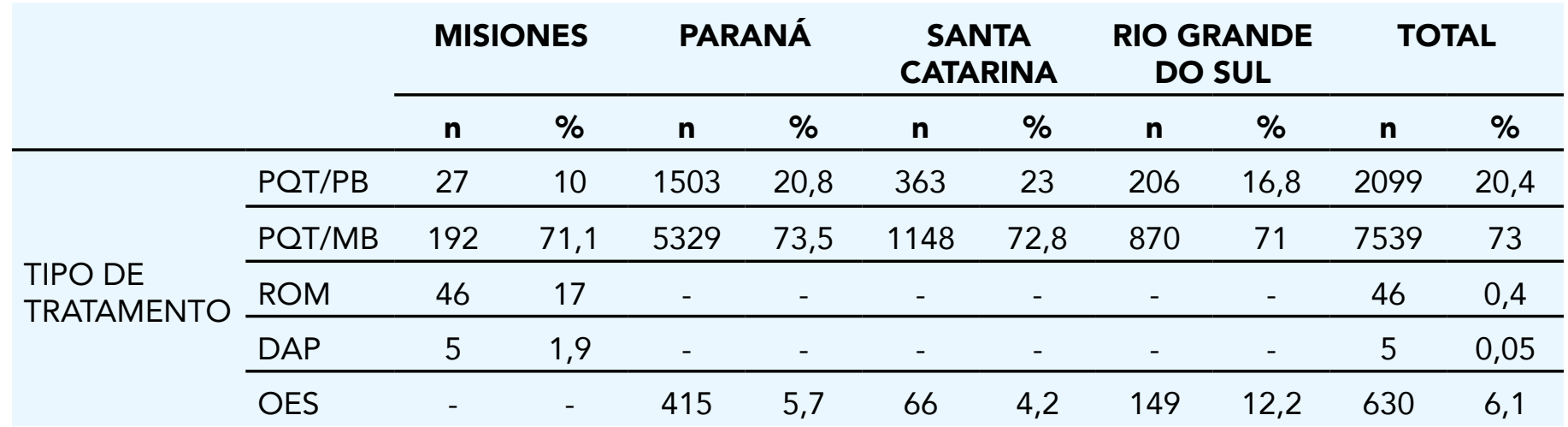

Legenda: PQT- Poliquimioterapia. PB- Paucibacilar. MB- Multibacilar. ROM- Rifampicina/Ofloxacina/Minociclina. DAP - Dapsona. OES - Outros esquemas substitutivos.

No período, notou-se uma situação hiperendêmica em 780 (65,5\%) dos municípios/ departamentos estudados. Destacando o estado do Paraná que apresentou $284(71,2 \%)$ dos municípios com taxas hiperendêmicas, com os municípios de Anahy, Rancho Alegre e Ramilândia obtendo as taxas de incidência mais elevadas, de 1395.68, 855.37 e 588.17 casos por 100 mil habitantes, respectivamente. Os dados do Rio Grande do Sul apresentaram $47(9,46 \%)$ municípios hiperendêmicos, sendo os mais acometidos Sede Nova (192 casos/100mil habitantes), Três Passos (187,72 casos/100mil habitantes) e Rolador (178,45 casos/100mil habitantes) (Figura 2). 


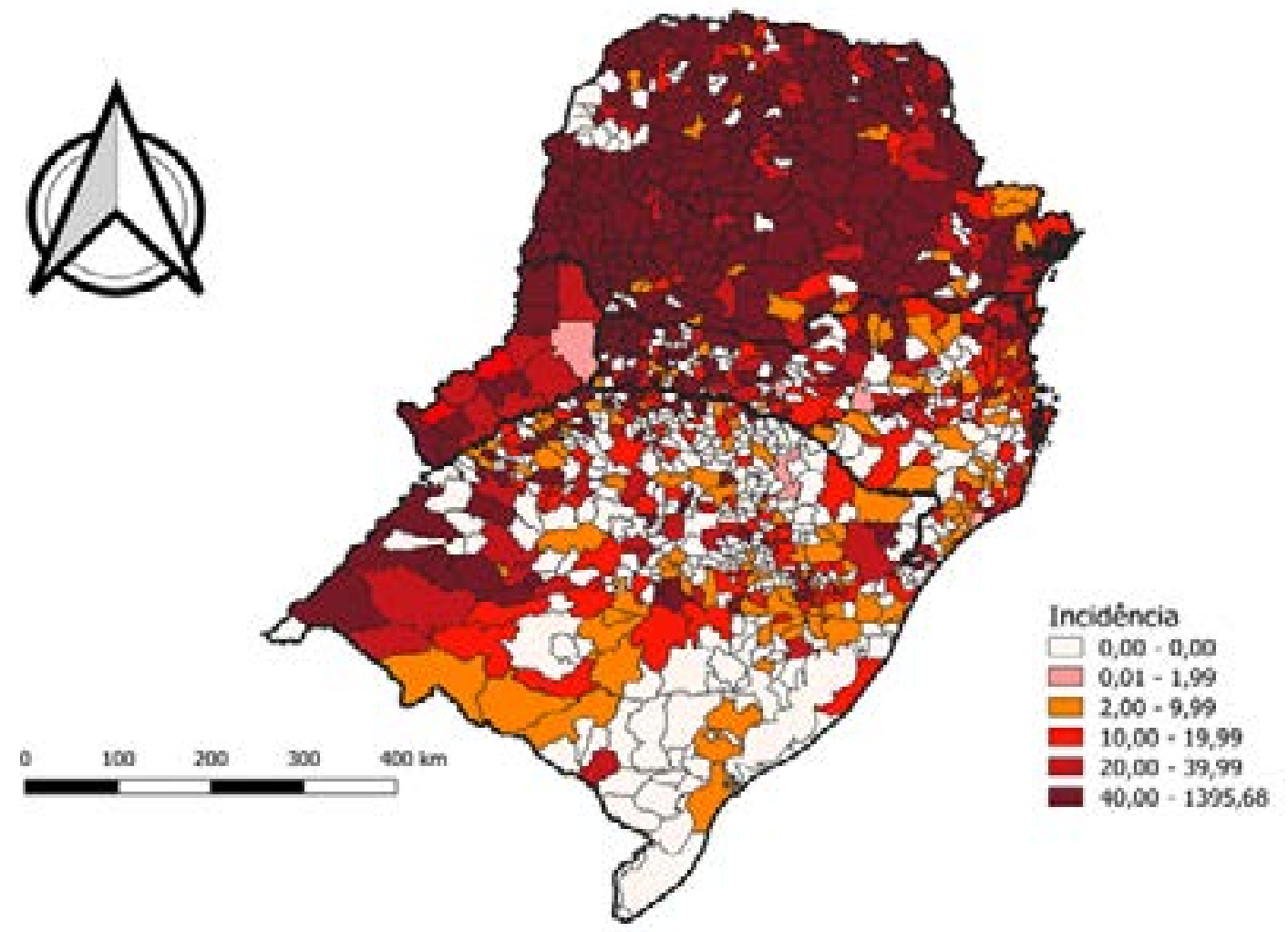

Figura 2- Taxa de incidência da hanseníase por 100 mil habitantes. Província de Misiones (AR), Paraná (BR), Santa Catarina (BR), Rio Grande do Sul (BR), 2010 a 2016

Nota: Classificação das taxas de incidência - baixa (menor que 2,00), média $(2,00$ a 9,99), alta $(10,00$ a $19,99)$, muito alta $(20,00$ a 39,99$)$ e situação hiperendêmica (maior ou igual a 40,00$)$.

O estado de Santa Catarina evidenciou 61 (20,68\%) municípios hiperendêmicos, sendo as taxas mais elevadas em Anchieta (274,88 casos/100 mil habitantes), Mondaí (234,26 casos/100 mil habitantes), e Dionisio Cerqueira (214,16 casos/100 mil habitantes).

A província de Misiones apresentou sete $(41,18 \%)$ departamentos hiperendêmicos, dando destaque aos departamentos de Eldorado (457,88 casos/100 mil habitantes), Puerto Iguazú (150,78 casos/100 mil habitantes) e Posadas (88,75 casos/100 mil habitantes), que obtiveram as maiores taxas de incidência.

Ainda referente à Figura 2, destacam-se os municípios onde a incidência no período permaneceu 0 (zero), mas estão rodeados ou próximos a municípios com altas taxas de incidências, como em RS e SC, ou ainda por município hiperendêmicos, sendo esta situação mais visível no PR. Municípios brasileiros sem registros de hanseníase rodeados de municípios hiperendêmicos ocorrem nos três estados brasileiros.

Quanto à análise espacial, a taxa de incidência na região Sul do Brasil apresentou associação espacial significativa com índice global I de Moran =0,3405 ( $p=0,001)$. $O$ detalhamento local da análise espacial é verificado pelo LISA, representado na Figura 3. 

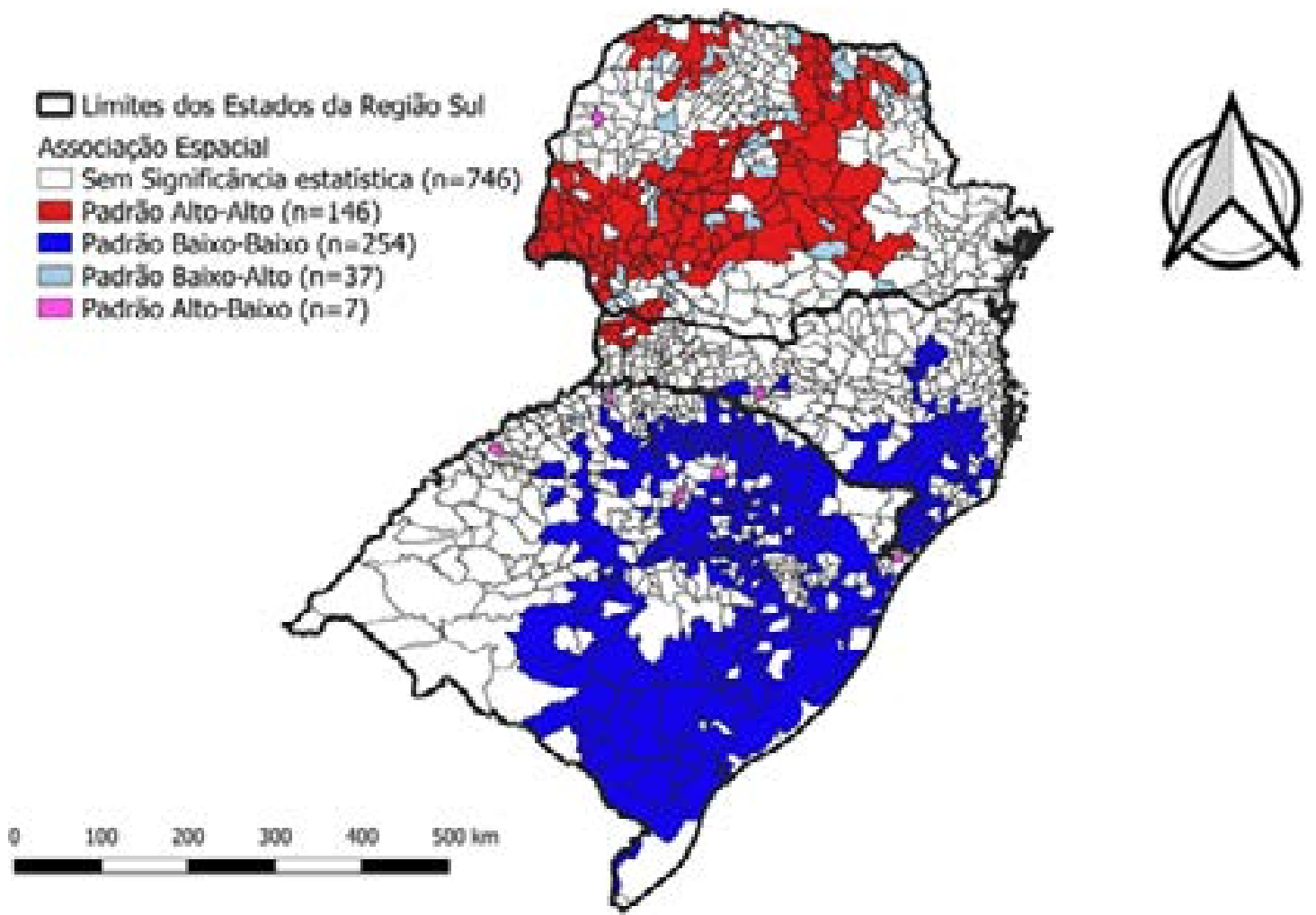

Figura 3 - Índice Local de Associação Espacial (LISA). Província de Misiones (AR), Paraná (BR), Santa Catarina (BR), Rio Grande do Sul (BR), 2010 a 2016

Na Figura 3, o estado do Paraná apresenta altos índices de correlação local Alto-Alto e Baixo-Alto, diferente do observado nos outros estados. As regiões destacadas na cor vermelha (Alto-Alto) apresentam incidência alta e seus vizinhos com valores altos também, e as regiões azuis claras (Baixo-Alto), apresentam regiões com baixa incidência, porém, cercado de regiões com altos índices.

O estado de Santa Catarina apresenta municípios com padrão Alto-Alto próximo à região de fronteira. E um cluster bastante extenso, com padrão Baixo-Baixo, é verificado entre municípios de Santa Catarina e do Rio Grande do Sul, ou seja, municípios com baixa incidência localizados próximos a municípios de baixa incidência. Contudo, cabe destacar alguns municípios com padrão Alto-Baixo (cor rosa), o que significa municípios com alta incidência cercado ou próximo a municípios de baixa incidência (Figura 3). Mesmo não sendo esse o padrão procurado por outros estudos, essa associação merece atenção quando se trata de um evento epidemiológico com a importância e características da hanseníase.

\section{DISCUSSÃO}

Como relatado em outros estudos ${ }^{(17)}$, esta pesquisa também mostrou maior número de casos de hanseníase nos homens, porém, com pouca diferença entre os sexos, como observado por outros pesquisadores ${ }^{(18-20)}$. Alguns autores afirmam que a maior exposição a ambientes de risco, principalmente nos locais de trabalho, contribui para elevar o número de casos nos homens e a preocupação com a estética corporal justificaria um grande número de casos do sexo feminino ${ }^{(20-21)}$.

Na população estudada ocorreu predomínio de pacientes multibacilares, recordando 
que é imprescindível a realização da classificação operacional dos casos de hanseníase, só assim é direcionada a forma de tratamento pela PQT. A classificação baseia-se no quantitativo de lesões cutâneas, sendo os pacientes $\mathrm{PB}$ os que apresentam até cinco lesões de pele com baciloscopia de raspado intradérmico negativo, e MB os que apresentam mais de cinco lesões ou baciloscopia de raspado intradérmico positiva. Sabendo que os doentes MB constituem o grupo de maior responsabilidade pela transmissão, devido à elevada carga bacilar(22), este estudo mostra a gravidade da situação em todas as regiões, pela grande quantidade de pacientes MB.

Entre as doenças transmissíveis, a hanseníase é uma das maiores causadoras de neuropatia periférica e incapacidade no mundo ${ }^{(23)}$, sendo uma das principais causas de incapacidades evitáveis, responsável por cerca de 3 milhões de pessoas incapacitadas em todo o mundo(24).

As incapacidades físicas na hanseníase estão relacionadas às sequelas e deformidades e indicam um diagnóstico tardio. Grande parte da população de estudo apresentou grau I já no momento do diagnóstico, indicando uma situação que afeta a vida de milhares de pessoas, porque compromete mecanismos de defesa, como a capacidade de sentir dor e tato, tornando-os mais vulneráveis aos riscos de acidentes, queimaduras, feridas entre outros $^{(25)}$.

No que se refere ao tratamento, desde 1940, a dapsona era usada isoladamente no tratamento da hanseníase e foi assim por cerca de três décadas. Porém, o uso prolongado, interrompido e inadequado da monoterapia com este medicamento leva ao desenvolvimento de casos resistentes à dapsona. A introdução do uso da clofazimina e da rifampicina abriu caminho para o uso combinado dos medicamentos e uma melhor resposta para eliminação do Mycobacterium leprae. Outras drogas, como a ofloxacina e a minociclina, também são usadas como drogas alternativas se algum medicamento da PQT for contraindicado(9).

Este estudo mostrou uma grande porcentagem de pacientes que utilizaram esquema de tratamento substitutivo, indicando intolerância medicamentosa aos fármacos da PQT/ OMS, o que resulta em um quadro preocupante. A classificação dos doentes e novas abordagens terapêuticas, incluindo a variação da duração e composição dos regimes, tem sido discutida. A vigilância desta endemia caminha para ser realizada dentro de um contexto integrado utilizando indicadores clinicamente relevantes, sendo o seguimento dos pacientes após alta indispensável para avaliação dos resultados terapêuticos ${ }^{(26)}$.

Como apresentado neste estudo, é possível observar muitos municípios com baixa incidência, rodeados de municípios hiperendêmicos, e, apesar da tendência de eliminação da hanseníase, essas disparidades regionais acabam auxiliando na manutenção da endemia. E embora, teoricamente, as ações de controle da hanseníase sejam implementadas em toda a rede de serviços públicos de saúde, uma das dificuldades evidenciadas é a detecção passiva dos casos. Há evidências de que, quando ocorre a busca ativa de casos, taxas inesperadamente maiores de incidência foram encontradas, mesmo em regiões bem desenvolvidas ${ }^{(4)}$.

As desigualdades socioeconômicas entre regiões são apontadas como os principais motivos dos altos índices da doença, porém, temos um Índice de Desenvolvimento Humano (IDH) de 0,756 na Região Sul do Brasil e de 0,829 na Província de Misiones, inclusive o estado do Paraná apontado pelo estudo como o mais endêmico está localizado no extremo socioeconômico dito favorável no Brasil, mostrando a complexidade da interação entre os determinantes de saúde ${ }^{(27)}$.

É importante lembrar que alguns territórios abordados englobam uma zona de fronteira, o que nos encaminha a uma ideia de ligação entre territórios e, para compreender, devemos considerar todo o conjunto territorial de ambos os países. Portanto, tratase de outra territorialidade, indo além das visões dos sujeitos, neste caso brasileiros e argentinos, com identidades diferenciadas ${ }^{(28)}$ e também dos riscos relacionados a esse convívio constante entre os países, pois, além do risco de migração de brasileiros doentes, 
ainda pode ocorrer a contaminação de argentinos no Brasil, e reciprocamente, devido a exposição ao bacilo. Tanto a análise de incidência quanto a análise de associação espacial demonstram áreas de risco na região fronteiriça do Brasil com a Argentina.

Cabe destacar que as interações transfronteiriças vão além da questão econômica, envolvendo todo um conjunto de interações materiais e não materiais, desde práticas econômico-comerciais até aquelas ocasionadas pela falta de serviços públicos ou privados, como na saúde, que geram constantes trânsitos entre um lado e outro da fronteira ${ }^{(28)}$.

Entre as limitações do estudo, é importante considerar o viés particular dos estudos ecológicos, a saber: as evidências da investigação não podem ser inferidas casuisticamente para o nível individual, sendo somente representativas para as populações estudadas. Ademais, a aquisição de informações por meio de banco de dados secundários pode trazer falhas concernentes ao processo de investigação/notificação ou digitação dos dados, que são alimentadas diretamente pelas unidades de saúde municipais.

\section{CONCLUSÃO}

O estudo mostrou que a Região Sul do Brasil e a Província de Misiones na Argentina apresentam alta endemicidade, transmissão ativa e diagnóstico tardio da hanseníase. Essas tendências, entrelaçadas à força de morbidade e de transmissão recente e persistente da doença, inclusive do diagnóstico tardio e provável endemia oculta pelo elevado percentual de casos multibacilares e casos com incapacidades visíveis, ampliam a relevância da hanseníase como problema de saúde pública na Região Sul do Brasil e na Província de Misiones Argentina.

Diante do cenário adverso das fronteiras, sugerimos o desenvolvimento de estudos em aglomerados espaciais, que incluam, além das ações de controle e vigilância estabelecida pelos Ministérios de Saúde dos países envolvidos, novas estratégias de busca ativa, campanhas e ações de educação visando diagnósticos e tratamento precoces.

Para isso, faz-se necessário um debate e iniciativas de políticas públicas diferenciadas para as zonas fronteiriças, para estabelecer uma agenda voltada ao fortalecimento da vigilância ativa da hanseníase. Essa agenda precisa envolver os preceitos do cuidado ético, humano e solidário, a fim de alcançar resultados realmente desejáveis para controle da hanseníase no mundo.

\section{REFERÊNCIAS}

1. Ma Y, Pei Q, Zhang L, Lu J, Shui T, Chen J, et al. Live Mycobacterium leprae inhibits autophagy and apoptosis of infected macrophages and prevents engulfment of host cell by phagocytes. Am J Transl. [Internet]. 2018 [acesso em 11 dez 2018]; 10(9). Disponível em: https://www.ncbi.nlm.nih.gov/pmc/ articles/PMC6176229/pdf/ajtr0010-2929.pdf.

2. Liu J, Wen Y, Xing Y, Wang S. Borderline tuberculoid leprosy mimicking sarcoidosis. Medicine [Internet]. 2018 [acesso em 01 nov 2018]; 97(32). Disponível em: https://doi.org/10.1097/MD.0000000000011616.

3. Sales OP, Martins FJ dos S, Amaral JB da LC. Hanseníase um problema de saúde pública no Tocantins: o que revelam os dados de domínio público de 2011 - 2015. Rev. Humanid. Inov. [Internet]. 2018 [acesso em 13 nov 2018]; 05(2). Disponível em: https://revista.unitins.br/index.php/humanidadeseinovacao/ article/view/593.

4. Vieira MCA, Nery JS, Paixão ES, Andrade VF de, Penna GO, Teixeira MG. Leprosy in children under 15 years of age in Brazil: A systematic review of the literature. PLoS Negl Trop Dis [Internet]. 2018 [acesso em 15 nov 2018]; 12(10). Disponível em: https://doi.org/10.1371/journal.pntd.0006788. 
5. Santos MDM dos. Incidência da hanseníase no Brasil. Facesa [Internet]. 2014 [acesso em 15 nov 2018]. Disponível em: https://www.senaaires.com.br/wp-content/uploads/2017/05/INCID\%C3\%8ANCIA-DAHANSEN\%C3\%8DASE-NO-BRASIL.pdf.

6. Ministério da Saúde (BR). Secretaria de Vigilância em Saúde. Coordenação Geral de Hanseníase e Doenças em Eliminação. Guia prático para operacionalização da Campanha Nacional de Hanseníase, Verminoses, Tracoma e Esquistossomose 2016. [Internet] Brasília: Ministério da Saúde; 2016 [acesso em 01 out 2018]. Disponível em: http://portalarquivos2.saude.gov.br/images/pdf/2017/fevereiro/11/GuiaOperacional-Campanha-2016-final.pdf.

7. Sociedade Argentina de Dermatologia. Resenha sobre la lepra. Ciudad de Buenos Aires-Argentina. [Internet]. 2016 [acesso em 05 nov 2018]. Disponível em: http://www.sad.org.ar/lepra/resenia.htm.

8. Odriozola EP de, Quintana AM, Gonzales V, Pasetto RA, Utges ME, Bruzzone AO, et al. Towards leprosy elimination by 2020: forecasts of epidemiological indicators of leprosy in Corrientes, a province of northeastern Argentina that is a pioneer in leprosy elimination. Mem. Inst. Oswaldo Cruz [Internet]. 2017 [acesso 01 out 2018]; 112(6). Disponível em: http://dx.doi.org/ 10.1590/0074-02760160490.

9. Naaz F, Mohanty PS, Bansal AK, Kumar K, Gupta UD. Challenges beyond elimination in leprosy. Int J Mycobacteriol. [Internet]. 2017 [acesso em 02 nov 2018]; 06(3). Disponível em: http://dx.doi.org/10.4103/ ijmy.ijmy 7017.

10. Organização Mundial da Saúde (OMS). Estratégia Global para Hanseníase 2016- 2020. Aceleração rumo a um mundo sem hanseníase. [Internet]. WHO; 2016 [acesso em 03 nov 2018]. Disponível em: http://apps.who.int/iris/bitstream/handle/10665/208824/9789290225201 pt.pdf?sequence=17.

11. Cardoso NA, Moura R. Regiões de fronteiras e fluxos migratórios: o caso do Paraná. In: Penha $B$, Desiderá Neto WA, Moraes RF de. O Mercosul e as regiões de fronteira. Rio de Janeiro; 2017. p. 53-99.

12. Daniel OJ. Adejumo OA, Oritogun KS, Omosebi O, Kuye J, Onyemaechi S, et al. Leprosy disease burden, active transmission and late presentation at the lowest administrative level in Nigeria: A spatial approach. Lepr Rev [Internet]. 2017 [acesso em 02 out 2018]; 88(3). Disponível em: https://pdfs. semanticscholar.org/c9e8/17e4bc2bbc92d3a986c0eed4823351f6ca70.pdf.

13. Wikiwand. Subdivisões na Argentina. [Internet]. 2018 [acesso em 15 nov 2018]. Disponível em: http:// www.wikiwand.com/pt/Subdivis\%C3\%B5es da Argentina\#/Ver tamb\%C3\%A9m.

14. Província de Misiones. Ubitaión Geográfica. [Internet]. 2017 [acesso em 10 nov 2018]. Disponível em: http://www.misiones.gov.ar/ubicacion-geografica/.

15. Sacardo DP. As peculiaridades dos sistemas de saúde dos países membros do Mercosul: perspectivas para a integração regional [tese]. São Paulo (SP): Universidade de São Paulo; 2009.

16. Instituto Brasileiro de geografia e Estatística (IBGE). Estado do Paraná - Brasil. [Internet]. 2018. [acesso em 05 agosto 2018]. Disponível em: https://cidades.ibge.gov.br/?lang=\&sigla=pr.

17. Campos MRM, Batista AVA, Guerreiro JV. Perfil Clínico-Epidemiológico dos Pacientes Diagnosticados com Hanseníase na Paraíba e no Brasil. Rev. bras. ciênc. saúde. [Internet]. 2018 [acesso em 01 out 2018]; 22(1). Disponível em: http://dx.doi.org/10.4034/RBCS.2018.22.01.11.

18. Goulart IMB, Arbex GL, Carneiro MH, Rodrigues MS, Gadia R. Efeitos adversos da poliquimioterapia em pacientes com hanseníase: um levantamento de cinco anos em um Centro de Saúde da Universidade Federal de Uberlândia. Rev. Soc. Bras. Med. Trop. [Internet]. 2002 [acesso em 13 out 2018]; 35(5). Disponível em: http://dx.doi.org/10.1590/S0037-86822002000500005.

19. Kubota RMM, Brancini VCL, Gouveia AS, Nardi SMT, Paschoal VD, Vendramini SHF. Efeitos adversos da poliquimioterapia para hanseníase: utilização de doses alternativas e avaliação pós alta. Hansen Int. [Internet]. 2014 [acesso em 01 nov 2018]; 39(1). Disponível em: http://www.ilsl.br/revista/detalhe artigo. php?id=12226.

20. Ribeiro Júnior AV, Vieira MA, Caldeira AP. Perfil epidemiológico da hanseníase em uma cidade 
endêmica no Norte de Minas Gerais. Rev Bras Clin Med. [Internet]. 2012 [acesso em 21 out 2018]; 10(4). Disponível em: http://files.bvs.br/upload/S/1679-1010/2012/v10n4/a3046.pdf.

21. Goiabera YNL de A, Rolim ILTP, Aquino DMC de, Soeiro VM da S, Inácio AS, Queiroz RC de S. Perfil Epidemiológico e clínico da hanseníase em capital hiperendêmica. Rev. enferm. UFPE [Internet]. 2018 [acesso em 08 out 2018]; 12(6). Disponível em: https://periodicos.ufpe.br/revistas/revistaenfermagem/ article/view/234693.

22. Ministério da Saúde (BR). Secretaria de Vigilância em Saúde. Guia Prático sobre a Hanseníase. [Internet] Brasília: Ministério da Saúde; 2017 [acesso em 03 out 2018]. Disponível em: http:// portalarquivos2.saude.gov.br/images/pdf/2017/novembro/22/Guia-Pratico-de-Hanseniase-WEB.pdf.

23. Faria L, Calábria LK. Aspectos históricos e epidemiológicos da hanseníase em Minas Gerais. Rev Med Saude Brasilia [Internet]. 2018 [acesso em 03 out 2018]; 06(3). Disponível em: https://portalrevistas.ucb. br/index.php/rmsbr/article/view/8394.

24. Souza CDF de, Fernandes TRM de O, Matos TS, Ribeiro Filho JM, Almeida GKA de, Lima JCB, et al. Grau de incapacidade física na população idosa afetada pela hanseníase no estado da Bahia, Brasil. Acta Fisiátr. [Internet]. 2017 [acesso em 15 nov 2018]; 24(1). Disponível em: http://dx.doi.org/10.5935/01047795.20170006.

25. Ministério da Saúde (BR). Secretaria de Vigilância em Saúde. Boletim Epidemiológico. [Internet] Brasília: Ministério da Saúde; 2018 [acesso em 02 out 2018]. Disponível em: http://portalarquivos2.saude. gov.br/images/pdf/2018/janeiro/31/2018-004-Hanseniase-publicacao.pdf.

26. Crespo MJ, Gonçalves A. Avaliação das possibilidades de controle da hanseníase a partir da poliquimioterapia. Rev. port. saude publica [Internet]. 2014 [acesso em 02 out 2018]; 32(1). Disponível em: http://dx.doi.org/10.1016/j.rpsp.2013.09.001.

27. Ribeiro MDA, Silva JCA, Oliveira SB. Estudo epidemiológico da hanseníase no Brasil: reflexão sobre as metas de eliminação. Rev. Panam. Salud Pública [Internet]. 2018 [acesso em 10 jan 2018]; 42(42).

Disponível em: https://doi.org/10.26633/RPSP.2018.42.

28. Ferrari M. Zona de fronteira, cidades gêmeas e interações transfronteiriças no contexto do MERCOSUL. Rev. transp. Territ. [Internet]. 2018 [acesso em 05 out 2018]; 09(1). Disponível em: http:// revistascientificas.filo.uba.ar/index.php/rtt/article/view/305/283.

29. Peiter, P. Encontro entre Saberes. Departamento de Saneamento e Saúde Ambiental da ENSP. [Internet]. 2007 [acesso em 05 nov 2018]. Disponível em: http://www.ensp.fiocruz.br/portalensp/informe/ site/materia/detalhe/5845.

Recebido: 14/02/2019

Finalizado: 09/09/2019

Autor Correspondente:

Keurilene Sutil de Oliveira

Universidade Estadual do Oeste do Paraná

R. Pompeu de Toledo, 333 - 85858-490 - Foz do Iguaçu, PR, Brasil

E-mail: keurisutil@gmail.com

Contribuição dos autores:

Contribuições substanciais para a concepção ou desenho do estudo; ou a aquisição, análise ou interpretação de dados do estudo - KSO, MAMA, AJS

Elaboração e revisão crítica do conteúdo intelectual do estudo - KSO, MAMA, JHD, AZ, RAAS

Aprovação da versão final do estudo a ser publicado - KSO, MAMA, JHD, AJS, AZ, RAAS

Responsável por todos os aspectos do estudo, assegurando as questões de precisão ou integridade de qualquer parte do estudo - KSO 